\title{
Synthesis of Functionalized Graphene/Polyaniline \\ Nanocomposites with Effective Synergistic Reinforcement on
}

\section{Anticorrosion}

Xinxin Sheng ${ }^{1}$, Wenxi Cai ${ }^{1}$, Li Zhong ${ }^{1}$, Delong Xie ${ }^{* 1,2}$, Xinya Zhang ${ }^{1}$

1. School of Chemistry and Chemical Engineering, South China University of Technology, Guangzhou, 510640, China

2. School of Chemical Engineering, Kunming University of Science and Technology, Chenggong Campus, Kunming, 650504, China 

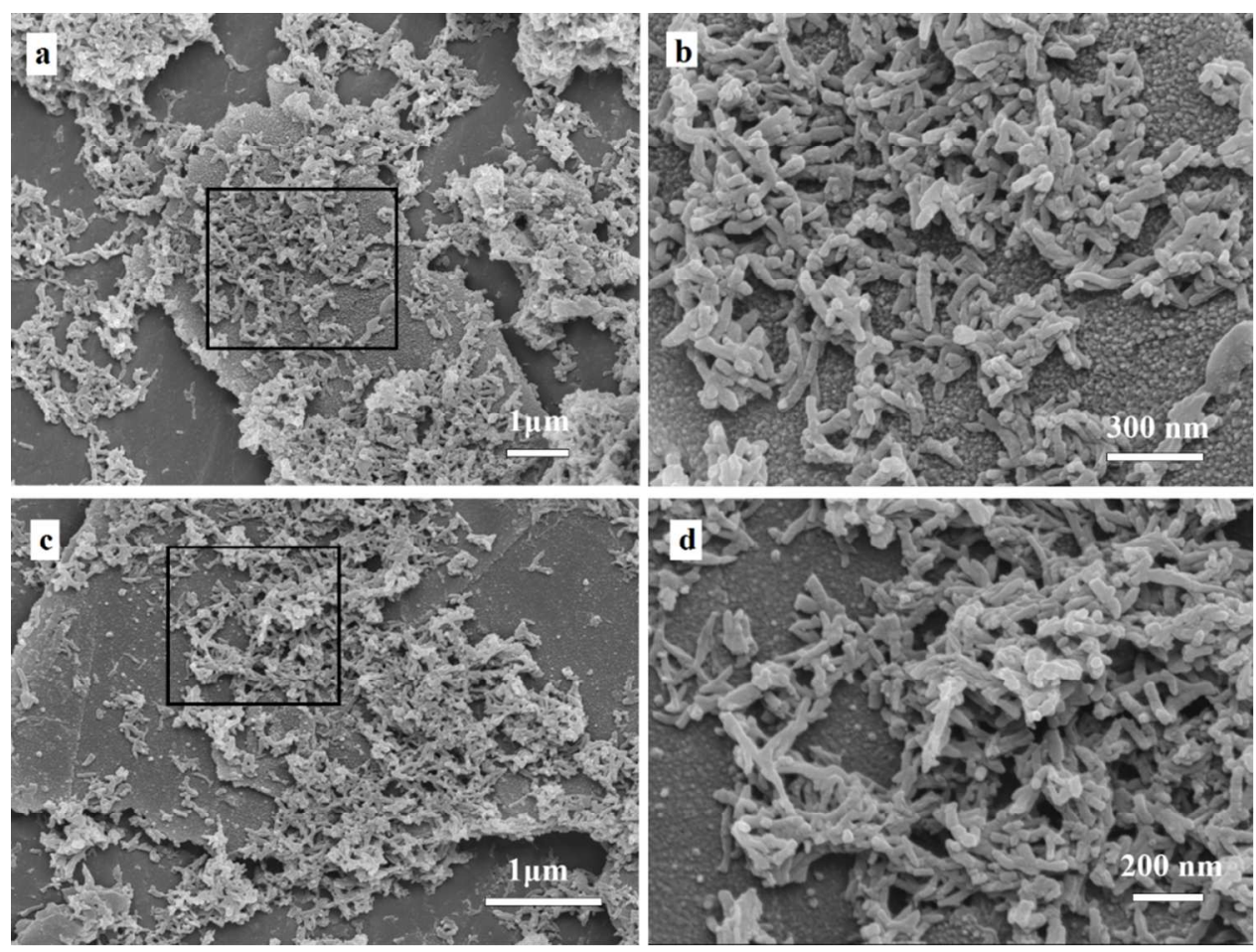

Figure S1. SEM image of PGO/PANI composites (a, c) at low magnification and (b, d) at high magnification

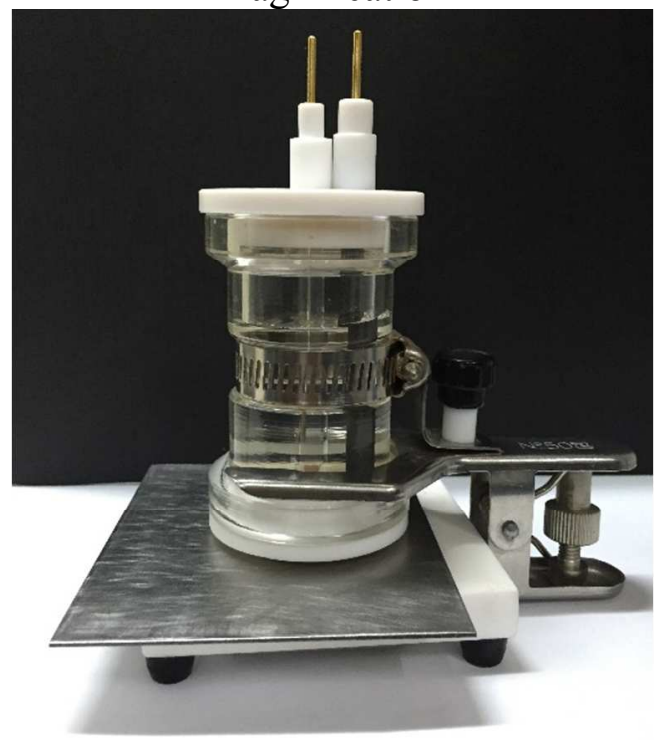

Figure S2. Assembly drawing of coating evaluation electrolytic cell. Carbon steel covered with coating film (area, $3.14 \times 1.47 \times 1.47 \mathrm{~cm}^{2}$ ) was used as working electrode, graphite electrode was used as counter electrode, and $\mathrm{Ag} / \mathrm{AgCl}$ was used as reference electrode. 